\title{
LA PERFORMANCE DELL'INSEGNANTE E IL RAPPORTO DI AFFETTIVITÀ E DI APPRENDIMENTO DAL PUNTO DI VISTA DI PIAGET E VALLONE
}

\section{RECENSIONE ARTICOLO}

DIAS, Adailton di Lauro ${ }^{1}$

DIAS, Adailton di Lauro. La performance dell'insegnante e il rapporto di affettività e di apprendimento dal punto di vista di Piaget e Vallone. Revista Científica Multidisciplinar Núcleo do Conhecimento. Ano 04, Ed. 07, Vol. 09, pp. 64-71. Iuglio 2019. ISSN: 2448-0959

\section{RIEPILOGO}

Lo studio affronta il tema del rapporto tra affettività e apprendimento. Cita l'importanza delle prestazioni dell'insegnante in questo contesto. Si rivolge a ciò che l'affettività, la cognizione e la motivazione collaborano allo sviluppo dell'apprendimento dello studente attraverso la mediazione degli insegnanti nella scuola quotidiana. Si basa su teoristi come Piatget e Vallone. Ha come obiettivo generale comprendere il rapporto tra apprendimento e affettività dal punto di vista di Piaget e Vallone. Porta come un problema di ricerca: Come può l'affettività, la cognizione e la motivazione collaborare allo sviluppo dell'apprendimento dello studente con la mediazione dell'insegnante in classe? Dagli studi condotti si tiene conto che, dal punto di vista di Piaget, nella sua teoria piagetiana, l'affettività, la cognizione e la motivazione sono condizioni necessarie nella costituzione dell'intelligenza e dell'apprendimento significativo. Secondo il Vallone, nella sua teoria chiamata vallone, per l'apprendimento, è necessario integrarsi tra insiemi funzionali (affettività, cognizione, motricità e persona).

\footnotetext{
${ }^{1}$ Mestrando em Ciências da Educação (Grendal University). Especialista em Língua Inglesa (FIJ). Graduado em Letras (UNEB). Graduado em Português e Inglês pela Universidade Metropolitana de Santos - SP. Professor EBTT Port/Ing - IFRR.
} 
Pertanto, è necessario sottolineare l'importanza delle relazioni consolidate con gli aspetti emotivi degli studenti nel processo di insegnamento e apprendimento. E che le relazioni che coinvolgono l'affettività possono influenzarsi a vicenda nel vostro comportamento, aiutandovi ad acquisire conoscenza più facilmente, rendendolo più intelligente, guidandolo e motivandolo ad azioni che coinvolgono l'apprendimento Significativo. Così, l'affettività, la cognizione e la motivazione aiutano nello sviluppo degli studenti di apprendimento nella vita scolastica quotidiana.

Parole chiave: affetto, apprendimento, insegnante, Piaget, Vallone.

\section{INTRODUZIONE}

Lo studio discute il rapporto tra apprendimento e affettività, evidenziando in questo processo l'importanza delle prestazioni dell'insegnante in classe, dimostrando che il suo stato emotivo, così come quello degli studenti riflette positivamente o negativamente nell'apprendimento. Porta anche il tema dell'affettività, della cognizione e della motivazione come fattori primari per lo sviluppo e l'apprendimento dello studente, dal punto di vista di Piaget e Wallon.

Il problema dello studio cerca di mettere in discussione: Come possono l'affettività, la cognizione e la motivazione collaborare allo sviluppo dell'apprendimento dello studente con la mediazione dell'insegnante in classe? Per raggiungere l'obiettivo generale che è configurato per comprendere il rapporto tra apprendimento e affettività dal punto di vista di Piaget e Vallone e anche per rispondere al problema di questo studio, è stata utilizzata la ricerca bibliografica. Le riflessioni sul rapporto tra l'apprendimento e lo sviluppo degli studenti hanno motivato la costruzione di questo lavoro. 


\section{L'AFFETTO, COGNITION E MOTIVATION COME RESPONSABILI PER LO SVILUPPO E LEARNING DELLO STUDENTE}

La affettività, la cognizione e la motivazione sono fattori essenziali per lo sviluppo e l'apprendimento degli studenti nella scuola quotidiana, anche per sviluppare l'intelligenza, dal momento che,

(...) senza affetto, non ci sarebbe alcun interesse, nessun bisogno, nessuna motivazione; e di conseguenza, domande o problemi non sarebbero mai posti e non ci sarebbe intelligenza. L'affettività è una condizione necessaria nella costituzione dell'intelligence (PIAGET, 1992, p. 32).

L'educazione occidentale ha come patrimonio culturale, la disconnessione dell'uomo delle sue emozioni, dei suoi pensieri, della propria realtà, che porta alla formazione di individui deboli di spirito, energia e emotivamente ignoranti. Si tratta, quindi, di una visione frammentaria dell'uomo, che purtroppo contribuisce a una rottura delle relazioni umane. Tuttavia, questi dualismi tradizionali del pensiero occidentale presenti nella storia della psicologia tradizionale sono stati confrontati e interrogati da teorie innovative con l'obiettivo di integrare, dialetticamente, la cognizione e l'affettività, la ragione e l'emozione.

Tuttavia, l'affettività è indispensabile per lo sviluppo intellettuale dei bambini e degli adolescenti, li spinge ad agire nell'oggetto della conoscenza. "Ma l'affettività non è nulla senza intelligenza, che fornisce loro i mezzi e chiarisce i fini" (PIAGET, 1992, p.70). A questo proposito, l'intelligenza agisce in affettivo, considerevolmente in tutto il processo dell'esistenza umana, in cui l'organizzazione quotidiana fornisce alla costruzione attiva, elementi favorevoli alla formazione della personalità.

Così, è possibile comprendere i rapporti tra affetto e intelligenza, questo corrisponde alle emozioni che si rivolgono al mondo esterno, orientate al mondo degli oggetti di studio e di osservazione. Ciò che si richiama in modo indaffarato è l'attenzione che il 
bambino e l'adolescente cominciano a dirigere verso le componenti del mondo che li circonda, per cercare di capire come funzionano le cose per adattarsi ad esse.

È l'interrelazione reciproca di questi due casi, affettivo e razionale, che si verifica la formazione e lo sviluppo della personalità, che qui è inteso come il prodotto del rapporto che si costruisce tra il soggetto e l'ambiente socioculturale, risultando in un essere unico, individuo.

Piaget (1971) ha avvertito che, nonostante la loro natura diversa, l'affettività e la cognizione sono inseparabili, perché in tutte le azioni simboliche e sensate appaiono indissociate. Ha documentato il comportamento efficace dell'azione e del pensiero come un aspetto cognitivo, attraverso strutture mentali, e un aspetto affettivo, attraverso un'energia, che è affettività.

Vygotsky (2002) comprende l'uomo come un essere che ha la capacità di pensare, ragione, deduzione e astratto, ma anche come qualcuno dotato di sentimenti, emozioni, desideri, immaginazione e sensibilizzazione al mondo esterno. Così, non è possibile analizzare il sentimento e la ragione separatamente. Piuttosto, un'analisi nella sua interezza ha senso, perché indica sovvenzioni favorevoli a possibili soluzioni sui problemi esistenti. Per "dimostra l'esistenza di un sistema dinamico di significati in cui si uniscono affettivo e intellettuale" (VYGOTSKY, 2002, p. 7). Tuttavia, è indispensabile rafforzare il significato delle relazioni che gli educatori stabiliscono con gli aspetti emotivi degli studenti nel processo di insegnamento e apprendimento.

Per quanto riguarda le emozioni, il Vallone (apud GALVàO, 1995) dice che è stato dall'inizio della vita; lo difende, come il primo e più forte legame tra gli individui. All'inizio della sua esistenza, il bambino diventa gradualmente un essere sociocognitivo durante la costruzione graduale, una visione unica e particolare della sua esistenza.

II wallon (apud GALVàO, 1995) afferma anche che l'affettività appare quando sorgono elementi simbolici, cioè si manifesta dal contatto con l'altro, rende chiaro che l'affettività comporta una serie di manifestazioni che comprendono sentimenti ed emozioni. 
All'interno di questa considerazione Galvào affronta nel testo "espressività ed emozioni, secondo la prospettiva del Vallone" che:

All'inizio, attraverso i suoi gesti impulsivi, contorsioni o spasmi del corpo, così come le espressioni emotive più primitive, come piangere o sorridere, il bambino umano mobilita le persone del suo ambiente in una sorta di contagio affettivo. L'adulto interpreta, di conseguenza, i suoi valori, i suoi desideri e le sue aspettative, il significato delle espressioni emotive del bambino, essendo portato ad agire secondo i suoi parametri culturali, i suoi desideri e le sue convinzioni individuali, avvolti nel clima di contagio proprio di questi (GALV, 1995, p. 74).

Tuttavia, in questo modo che il bambino umano deve esprimersi sono manifestazioni di pura emozione e provocare reazioni affettive che li inducono a soddisfare i bisogni del bambino. In questo senso, l'affettività è il comportamento in grado di influenzare l'altro, in modo che lo guida in azione. La prima forma di affetto sta nelle emozioni, il cui ruolo è quello di unire gli individui tra loro da reazioni organiche e intime in modo globale e indifferenziato.

È attraverso questa unione che gli studenti quando arrivano a scuola si relazionano tra loro e guadagnano progressi significativi nella sfera cognitiva. Con questo, i legami affettivi si espandono e l'insegnante svolge un ruolo importante nello sviluppo degli studenti nel contesto scolastico.

Per aderire al comportamento motivazionale agli aspetti affettivi e cognitivi, vale la pena ricordare che:

Lo sviluppo dell'intelligenza permette senza dubbio che la motivazione sia risvegliata da un numero crescente di oggetti o situazioni. Tuttavia, nel corso di questo sviluppo, il principio di base rimane lo stesso: l'affettività è la molla trainante delle azioni, e la ragione è al suo servizio (LA TAILLE, 1992, p. 65). 
La motivazione, a sua volta, è parte integrante del processo di insegnamento e apprendimento, è uno degli elementi considerevoli ed essenziali per imparare o realizzare qualcosa. Tutte le azioni sono mosse da una forza motivazionale. "La motivazione è la forza motrice della condotta. È la condizione interna che attiva l'individuo e lo predispone a rilasciare determinate risposte" (CORIA-SABINI, 2004, p. 83).

Tuttavia, per imparare o insegnare, deve verificarsi una forza motrice motivazionale. L'assenza di questo giorno per giorno nella pratica educativa sia nello studente e nella direzione e nella facoltà comprometterà lo sviluppo cognitivo degli studenti.

\subsection{RAPPORTO AFFETTIVO E ITS CON L'APPRENDIMENTO: LE PRESTAZIONI DELL'INSEGNANTE NELLA CLASSE}

Comprendendo l'importanza dell'azione degli insegnanti in classe, si percepisce che il suo stato emotivo, così come quello degli studenti, si riflette nell'apprendimento. L'insegnante mostrando affetto nelle sue azioni, motiva lo studente a voler partecipare attivamente alle sue classi, percependo l'insegnante come un amico sempre pronto ad aiutarlo e insegnarlo.

Per Oliveira e Chadwick (2001, p. 52),

Lo stato emotivo e affettivo degli studenti influenza il loro apprendimento. La motivazione è come un trigger che guida l'apprendimento e stabilisce le condizioni in cui si verifica. Le emozioni svolgono un ruolo importante nell'apprendimento in qualsiasi fase della vita. Ma sono particolarmente importanti negli studenti più giovani, in quanto la situazione scolastica è abbastanza artificiale rispetto alla loro vita e non molto compatibile con le loro preferenze.

La pratica di un insegnante affettivo sarà quella di rispettare il ritmo di apprendimento di ogni studente, perché ha la sensibilità di percepire quando o non procedere con il contenuto. Uno studente potrebbe aver bisogno di più tempo per imparare una certa 
disciplina rispetto al suo collega, ma può anche essere più veloce in una disciplina che ha più affinità.

Come afferma Mendes (2017), è necessario riflettere sulla pratica pedagogica e sulla formazione degli insegnanti. Egli afferma che l'istruzione continua riflette sul ruolo dell'educatore come professionista, tuttavia questa formazione riflette, soprattutto, in questo professionista come persona, perché gli individui finiscono per essere influenzati da altre persone e da ciò che imparano in decisioni o scelte e ciò che trasmetterà nella scuola di tutti i giorni. La teoria scelta da questo autore è murale, il cui punto principale è quello di integrare insiemi funzionali - affettività, cognizione, motricità e la persona e anche l'organismo centrale.

Così, per il Vallone (2007), questo fornisce la comprensione della persona nella sua interezza, comprendendola non solo dal punto di vista di uno dei set, ma attraverso la continua integrazione tra di loro. Lo studio di Henri Wallon (1999) sottolinea anche l'ambiente in cui la persona è inserita, perché sia l'individuo che la persona subisce influenza l'uno dall'altro. Da questo punto di vista, il Vallone considera questo elemento altrettanto importante nel suo processo di sviluppo.

Affinché l'insegnante svolgi un buon lavoro, con l'obiettivo di raggiungere il maggior numero possibile di studenti, ha bisogno di utilizzare varie tecniche di insegnamento, adottando non solo le singole opere, ma anche in coppia, gruppo, ecc. Inoltre, è anche importante che gli insegnanti abbiano un buon rapporto con gli altri insegnanti della scuola, per scambiare informazioni sugli studenti all'interno di ogni area di conoscenza.

In questo contesto l'insegnante è incaricato di promuovere i mezzi che garantiscono la motivazione, in cui porterà all'apprendimento. A proposito di questo Coria-Sabini indirizzi:

La motivazione ha un aspetto ciclico. In primo luogo, c'è un bisogno che si manifesta attraverso una specifica stimolazione interna. In secondo luogo, vi sono azioni volte a raggiungere un obiettivo. Una volta raggiunto questo obiettivo, vengono seguiti i soccorsi e la diminuzione della 
tensione. Questo sollievo è temporaneo e dopo qualche tempo il ciclo riprende. Un bambino che piange si calma dopo l'allattamento. Tuttavia, ore dopo la fame riappare e dovrebbe essere risatisfiedato (CORIASABINI, 2004, p. 84).

In realtà questo è ciò che dovrebbe accadere in classe, l'insegnante deve avere strategie che motivano lo studente ad agire sull'oggetto della conoscenza; quando lo studente riesce a trarre le sue conclusioni si sentirà sollevato. Da questo è colui che entra di nuovo l'insegnante portando nuove problematiche e lo studente andrà ancora una volta alla ricerca della nuova conoscenza. Ogni volta che lo studente raggiunge I'obiettivo proposto, spetta all'insegnante proporre altre sfide per lo studente continuare a sviluppare il loro potenziale.

Per quanto riguarda i mezzi o le metodologie che l'insegnante utilizza per motivare i suoi studenti non vengono al caso, ognuno funziona in modo differenziato, ciò che conta è lavorare in un modo che lo studente percepisce l'apprendimento come un risultato personale. Come, per esempio, uno studente svolge una certa attività che ha ritenuto incapace di adempiere, dopo che l'insegnante gli ha offerto un brindisi sfidandolo a eseguirlo. Con questo, lo studente può trarre le sue conclusioni che è in grado di svolgere l'attività senza bisogno di premi, ma come un risultato personale.

Tuttavia, gli approcci degli autori citati, come Piaget, Vygotsky e Wallon dimostrano come l'emozione accanto alla ragione svolga un ruolo essenziale nell'apprendimento degli studenti. Secondo loro, il lavoro dell'insegnante non può limitarsi alla mera memorizzazione, ma sentire ed emozionare con loro. Purtroppo, questa comprensione della rilevanza e del ruolo adottati dalle emozioni nel processo di insegnamento e apprendimento è rara tra gli insegnanti, poiché le informazioni che guidano questa idea non vengono trasmesse, e sempre tenendo conto che le teorie educative, come il tecnicismo, che sono alla base della pratica pedagogica, non danno il giusto valore a questo aspetto emotivo dello sviluppo umano. 
Saltini cita nuovi paradigmi nell'educazione, tra cui, vale la pena menzionare ciò che dice:

Ci sarà uno spazio e un tempo all'interno della scuola in cui potrò evidenziare i miei compagni di classe, il mondo in cui vivo, le mie idee, fantasie, le mie scoperte, invenzioni, desideri e sogni. Questi, che emergono dal nostro nucleo, legano e hanno senso negli oggetti del mondo esterno, cioè ognuno dentro di sé costruisce il proprio legame, creando i propri simboli e valori, così come il loro significato speciale, ed è attraverso questi stessi significati che il soggetto crea collegamenti tra il mondo interiore. Da questo e con questo dobbiamo rispettare e creare una metodologia pedagogica che porti il soggetto al suo pieno sviluppo (SALTINI, 2008, p. 31).

In questo senso, ci si aspetta che la scuola diventi un ambiente di indagine, in cui il soggetto di questo processo possa esprimere le sue forme di pensiero, i suoi dubbi e le sue scoperte, in modo che migliori la sua capacità comunicativa e si espanda, significativamente, il suo inserimento nello spazio che sviluppa il potenziale degli studenti non solo nel campo della razionalità, ma anche nel campo delle emozioni.

In breve, Piaget, oltre a dare priorità all'importanza della maturazione biologica per l'acquisizione dell'apprendimento, ha anche considerato l'importanza dell'interazione per la costruzione della conoscenza. Vigotsky aveva tutta la sua teoria supportata nell'interazione tra individuo e mezzo. Affinché lo scambio tra pari sia più efficace, ha sottolineato il ruolo del linguaggio per l'espressione del pensiero. Concentrandosi sul rapporto tra domini affettivi e cognitivi, Wallon ha creato la teoria dello sviluppo della personalità, evidenziando che questo si forma tra l'affettività e la cognizione. Inoltre, ha sottolineato che l'affettività è una delle fasi più antiche dello sviluppo.

\section{CONCLUSIONE}

Lo studio sottolinea la necessità dell'insegnante di cercare in classe, l'integrazione tra 
affettiva e cognitiva nel processo di insegnamento e apprendimento, poiché l'affettività e la cognizione sono interconnesse. In questo contesto, lo sviluppo della precisa affettività delle elaborazioni effettuate nel piano di intelligence, e anche, lo sviluppo dell'intelligenza ha bisogno delle costruzioni affettive.

L'affettività è ancora un ampio campo di ricerca, è stato notato attraverso questo studio che è estremamente importante per il processo educativo e soprattutto per stabilire un buon rapporto tra insegnante e studente.

Purtroppo, durante il loro sviluppo, i bambini spesso crescono in un ambiente in cui le persone non si rispettano, vivendo in ostilità, dove l'affettività è assente. La tendenza è che questi bambini diventino aggressivi sia nelle loro famiglie che a scuola. Dal momento in cui i bambini diventano aggressivi, sorgeranno difficoltà nei rapporti sociali. Pertanto, è necessario che l'insegnante sappia come trattare con i suoi studenti dimostrando affetto nelle sue azioni.

Tuttavia, l'educatore dovrebbe essere consapevole che non è sufficiente essere solo affettivo per lo studente di imparare. Per questo, l'insegnante deve, prima di tutto, essere un ricercatore, preoccupato per la sua pratica pedagogica, impegnato nella sua funzione sociale ed etica nelle sue azioni.

L'affettività è solo un percorso per migliorare il rapporto insegnante-studente, che contribuirà al successo scolastico di entrambi. È attraverso l'affetto al momento del dialogo tra tutti i membri della comunità scolastica che i dubbi possono essere sattati, l'indisciplina può essere aggirata e il desiderio di apprendimento dello studente sarà alto e l'apprendimento raggiunto.

\section{RIFERIMENTI}

CORIA-SABINI, Maria Aparecida. Psicologia do Desenvolvimento. 2. ed. São Paulo: Ática. 2004. 
GALVÃO, Izabel. Henri Wallon: Uma concepção dialética do desenvolvimento infantil. Petrópolis, RJ: Vozes, 1995.

LA TAILLE, Yves de; OLIVEIRA, Marta Kohl de; DANTAS, Heloysa. Piaget, Vygotsky e Wallon: teorias psicogenéticas em discussão. 13. ed. São Paulo: Summus, 1992.

MENDES, Daniela Barros. Memórias afetivas: A constituição do professor na perspectiva de Henri Wallon. Sao Paulo: Loyola, 2017.

PIAGET, Jean. A equilibração das estruturas cognitivas. Problema central do desenvolvimento. Trad. Álvaro Cabral. Rio de Janeiro: Zahar, 1976.

. Desenvolvimento e aprendizagem. In: BRINGUIER, J.; PIANCELLA, J.R NESS. J.S.V., Org. In: Studying teaching. 2. ed. Prentice-Hall, 1971.

SALTINI, Cláudio J. P. Afetividade e Inteligência. 5. ed. - Rio de Janeiro: Wak Editora. 2008.

VYGOTSKY, Lev Semenovich. A formação social da mente: o desenvolvimento dos processos psicológicos superiores. 6. ed. São Paulo: Martins Fontes, 2002.

WALLON, Henri. A evolução psicológica da criança. Lisboa: Edições 70, 1999. . Afetividade e aprendizagem - Contribuições de Henry Wallon. São Paulo: Edições Loyola, 2007.

Inviato: giugno 2019.

Approvato: luglio 2019. 